\title{
Integrating vectors for genetic studies in the rare Actinomycete Amycolatopsis marina
}

\author{
Hong Gao ${ }^{1,3^{*}} \mathbb{D}$, Buvani Murugesan', Janina Hoßbach', Stephanie K. Evans', W. Marshall Stark ${ }^{2}$ \\ and Margaret C. M. Smith ${ }^{1}$
}

\begin{abstract}
Background: Few natural product pathways from rare Actinomycetes have been studied due to the difficulty in applying molecular approaches in these genetically intractable organisms. In this study, we sought to identify more integrating vectors, using phage int/attP loci, that would efficiently integrate site-specifically in the rare Actinomycete, Amycolatopsis marina DSM45569.

Results: Analysis of the genome of A. marina DSM45569 indicated the presence of attB-like sequences for TG1 and R4 integrases. The TG1 and R4 attBs were active in in vitro recombination assays with their cognate purified integrases and attP loci. Integrating vectors containing either the TG1 or R4 int/attP loci yielded exconjugants in conjugation assays from Escherichia coli to A. marina DSM45569. Site-specific recombination of the plasmids into the host TG1 or R4 attB sites was confirmed by sequencing.

Conclusions: The homologous TG1 and R4 attB sites within the genus Amycolatopsis have been identified. The results indicate that vectors based on TG1 and R4 integrases could be widely applicable in this genus.
\end{abstract}

Keywords: Rare Actinomycetes, Amycolatopsis, Integrating vectors, TG1 integrase, R4 integrase

\section{Background}

Streptomyces bacteria are widely exploited for their abundant bioactive natural products [1]. However, after decades of exploitation, the rate of discovery of new Streptomyces-derived bioactive products has declined, and interest has grown in other potential non-Streptomycete sources, such as the rare Actinomycetes $[2,3]$.

Amongst rare Actinomycetes, the Amycolatopsis genus is of particular interest for its production of critically important antibiotics such as vancomycin [4] and rifamycin [5], as well as a diverse range of active natural products [6-8]. The publicly available NCBI database contains nearly 90 genomes of Amycolatopsis strains, covering more than 40 species from this genus. Similar to

\footnotetext{
*Correspondence: gaohong221@gmail.com; h.gao@tees.ac.uk

'Department of Biology, University of York, York, North Yorkshire YO10 5DD, UK

${ }^{3}$ Present address: School of Science, Engineering \& Design, Teesside University, Middlesbrough TS1 3BX, UK

Full list of author information is available at the end of the article
}

Streptomyces, the genome of each Amycolatopsis contains averagely over 20 secondary metabolic gene clusters [9]. The mining of these metabolic clusters offers excellent potential for novel antibiotic discovery.

Phage-encoded serine and tyrosine integrases catalyse site-specific integration of a circularised phage genome into the host chromosome as part of the process to establish a lysogen. DNA integration mediated by serine integrases occurs between short (approximately $50 \mathrm{bp}$ ) DNA substrates that are located on the phage genome (the phage attachment site attP), and the host genome (the bacterial attachment site $a t t B$ ). The product of attP $\mathrm{x}$ att $B$ recombination is an integrated phage genome flanked by two new sites, attL and attR, each of which contains half-sites from $a t t P$ and $a t t B$. During phage induction, integrase in the presence of a recombination directionality factor (RDF) again mediates site-specific recombination, but this time between attL and attR, to excise the phage genome, which can then be replicated during a lytic cycle. The mechanism of recombination

(c) The Author(s). 2019 Open Access This article is distributed under the terms of the Creative Commons Attribution 4.0 International License (http://creativecommons.org/licenses/by/4.0/), which permits unrestricted use, distribution, and reproduction in any medium, provided you give appropriate credit to the original author(s) and the source, provide a link to the Creative Commons license, and indicate if changes were made. The Creative Commons Public Domain Dedication waiver (http://creativecommons.org/publicdomain/zero/1.0/) applies to the data made available in this article, unless otherwise stated. 
and the factors that control integration versus excision have been elucidated in recent years [10-12].

Integrating vectors based on the Streptomyces phage $\phi C 31$ integrase and attP locus are best known and most widely used in Actinomycete genome engineering [1316], and in addition to the phage recombination machinery (int/attP), integrating vectors contain a replicon for maintenance in Escherichia coli, an oriT for conjugal transfer and a marker or markers for selection in E. coli and the recipient. They are powerful genome engineering tools that act in an efficient, highly controllable and predictable way [17].

Using serine integrase-mediated recombination, these integrating vectors require no additional phage or host functions for integration, which is an especially important feature when they are used in other organisms that cannot be infected by the phages. This property makes serine integrase-based vectors promising tools for use in various systems $[10,18]$. However, the use of these integration vectors has not been fully explored in rare Actinomycetes, e.g. Amycolatopsis. There is one reported example of a conjugation system based on $\phi C 31$ integrase in Amycolatopsis japonicum MG417-CF17 [19], and it has been reported that other Amycolatopsis species lack $\phi C 31$ attB sites in their chromosomes [20]. The фBT1 attB sites have been more commonly identified in Amycolatopsis. A vector based on $\phi \mathrm{BT} 1$ int/attP has been successfully transferred into Amycolatopsis mediterranei [21]. Furthermore, electroporation remains the most widely applied method for transfer of integrative plasmids into this genus, rather than conjugation $[20,21]$.

In this paper, we chose to study A. marina DSM45569, a species isolated from an ocean-sediment sample collected in the South China Sea [22]. Since the marine environment has been assumed to offer an as yet mostly untapped treasure of chemical biodiversity [23], we are quite interested in natural product discovery from $A$. marina. We explored the application of bacterial genetic engineering using serine integrases and developed conjugative and integrating vectors for use in this species. We present evidence suggesting that these vectors could be applied to other species in this genus, thus opening up the prospect for versatile genetic manipulation of Amycolatopsis.

\section{Results}

Identification of attB-like sequences from the genome of A. marina DSM45569

The primers used in this study were listed in Table 1. The sequences of $a t t B$ sites recognised by a variety of integrases ( $\phi C 31$ [24], $\phi$ Joe [25], Bxb1 [26], R4 [27], SPBC [28], SV1 [29], TG1 [30] and TP901 [31]) were used in BLAST searches of the genome sequence of $A$. marina DSM45569 (NCBI Genome Database
NZ_FOKG00000000). The most significant hits for R4 and TG1 attB sites had the highest identities and lowest $E$-value (Table 2). The recognised R4 attB-like site is located within a gene predicted to encode a fatty-acyl-CoA synthase (SFB62308.1), and the TG1 attB site is located within a gene predicted to code for a putative succinyldiaminopimelate transaminase (WP_091671332.1). The BLAST analysis was extended to other species of Amycolatopsis to assess the conservation of these attB sites in the genus (Fig. 1). Both R4 and TG1 attB sites were highly conserved relative to the $a t t B$ sites originally identified from Streptomyces parvulus [32] (84\% for R4 integrase) and Streptomyces avermitilis [30] (62\% for TG1 integrase).

\section{A. marina attB-like sequences for TG1 and R4 are both active in in vitro recombination}

In each recombination reaction, substrates containing attP and the putative $a t t B$ site were mixed in cognate pairs with different concentrations of purified R4 or TG1 integrase in the corresponding buffer and incubated overnight at $30^{\circ} \mathrm{C}$, as described in Methods. The expected recombination events and the nature of the products are shown in Fig. 2a. TG1 catalysed recombination between the substrates more efficiently than R4 (Fig. 2b). As expected because neither phage is an Amycolatopsis phage, the recombination efficiencies for each integrase were observably better when the Streptomyces attB sites were used (Fig. 2c) compared to the A. marina $a t t B$ sites (Fig. 2b), particularly for TG1 integrase. Nevertheless, the presence of recombination activity indicated that both $A$. marina attB sites were functional and were likely to be active integration sites for integrative conjugation vectors.

\section{In vivo integration}

A. marina DSM45569 is unable to grow in the presence of apramycin, so integrating plasmids pHG4 and pJH1R4, containing the apramycin resistance determinant $\operatorname{aac}(3) I V$, were constructed. Following the standard Streptomyces conjugation protocol (see Methods), a frequency of approximately 160 exconjugants $/ 10^{8}$ spores was obtained for the transfer of pHG4 (encoding TG1 integrase), while the conjugation efficiency of pJH1R4 (R4 integrase) was only 20 exconjugants $/ 10^{8}$ spores (Table 3). For each integration, six exconjugants were picked at random and streaked on SM (soya mannitol) agar containing apramycin. Genomic DNA was then prepared and used as the template in PCR reactions, in which the primer pairs of TG1-attL-Am-for/rev and $\mathrm{R} 4$-attL-Am-for/rev were used to test for the occurrence of recombination at the expected TG1 and R4 attB sites (Fig. 3). All PCR reactions using exconjugants as templates gave the expected band sizes. Sequencing (GATC, Germany) of the PCR products with the primers 
Table 1 Oligonucleotides used in this study

\begin{tabular}{|c|c|}
\hline Oligonucleotide & Sequence $\left(5^{\prime}-3^{\prime}\right)$ \\
\hline pHG1A-for & CGAACGCATCGATTAATTAAGGAGGATCGTATGACGACCGTTCCCG \\
\hline pHG1A-rev & CGTGGTGGGCGCTAGCCTCCTCTAGTCATCCGTCG \\
\hline pHG1-for & ACTAGAGGAGGCTAGCTTCAATGGAGGAGATGATCGAGG \\
\hline pHG1-rev & GCAGGTCGACTCTAGATCTCGCTACGCCGCTACG \\
\hline pHG4-for & CGAACGCATCGATTAATTAAGCGGCCGCCATATGGAATTCGGTACCGCATGCAGATCTAGGAACTTCGAAGTTCCCGC \\
\hline pHG4-rev & TGATTACGCCAAGCTTTCGACTCTAGAGTAAGCGTCACGG \\
\hline pJH1R4-for & CTAGCGATTGCCATGACGTCGGAGCTGCTTACCAATGTC \\
\hline pJH1R4-rev & AAGAGGCCCGCACCGATTCCAAGAGGCCGGCAACTAC \\
\hline TG1-attB-Am-for & TCGATCTCCAGTGCGGGCAAGACGTTCAACTGCACCGGCTGGAAGATCGGGACCACCGGACGAACGCA \\
\hline TG1-attB-Sa-for & TCGATCAGCTCCGCGGGCAAGACCTTCTCCTTCACGGGGTGGAAGGTCGGCGGTGGAGCTCGGAGA \\
\hline R4-attB-Am-for & GGTTGCCCATCACCATGCCGAAGCAGTGATAGAAGGGAACCGGGATGCAGGTGAGAAGGTGCTCGTGT \\
\hline R4-attB-Sp-for & AGTTGCCCATGACCATGCCGAAGCAGTGGTAGAAGGGCACCGGCAGACACGGTGAGAAGGTGCTCGTGT \\
\hline attB-rev & CTGCATCTCAACGCCTTCCGG \\
\hline TG1-attP-for & AACCTTCACGCTCATGCC \\
\hline TG1-attP-rev & GTCGAGATTCTCCGTCTCCTG \\
\hline R4-attP-for & GATCGGTCTTGCCTTGCTC \\
\hline R4-attP-rev & ACCCGCAGAGTGTACCCA \\
\hline TG1-attL-Am-for & ACAACCCCACCGGCACCGTCTTCA \\
\hline TG1-attL-Am-rev & AGTATAGGAACTTCGAAGCAGCTC \\
\hline R4-attL-Am-for & CGGCCGGTGATGTTGACGT \\
\hline R4-attL-Am-rev & TCGGCCGTCACGATGGTCA \\
\hline
\end{tabular}

The attB sequences are shown underlined

TG1-attL-Am-for and R4-attL-Am-for confirmed that the plasmids had integrated into the predicted $a t t B$ sites for TG1 or R4 integrase within A. marina DSM45569 (Fig. 4).

\section{Discussion}

The lack of effective genetic engineering tools is considered one of the greatest hindrances in the search for new natural products from rare Actinomycetes [33-35]. Previous studies in rare Actinomycete species have focused mainly on the use of the well-characterised $\phi C 31$-based integration vectors, and have mostly overlooked tools based on other phage integrases [36-38]. Additionally, the easy-handling conjugation methods used widely in Streptomyces gene transfer have shown little success in rare Actinomycetes, including species in the genus Amycolatopsis, so direct transformation with plasmids [39-41], or electroporation, has been the long-preferred method of gene transfer for species in this genus [5, 42-44]. However, the growing interest in the use of serine integrases for synthetic biology applications [10] has led to further research into expanding the pool of available enzymes and their potentials as genetic tools [45-47]. Therefore, within this study, we explored whether integrating vectors based on eight serine integrases could be employed for the genetic engineering of $A$. marina DSM45569. Sequence analysis of the $A$. marina DSM45569 genome identified close matches to the $a t t B$ sites used by TG1 and R4 integrases. Although conjugation frequencies were relatively low, integrating plasmids based on the TG1 and R4 recombination systems have been successfully integrated into the expected $a t t B$ sites in $A$. marina DSM45569. Conservation between the $a t t B$ sites for TG1 and R4 in a number of Amycolatopsis species is high, suggesting that plasmids with the integration systems from these phages should be widely useful in this genus, including the species which have garnered much interest as natural product producers, such as Amycolatopsis balhimycina [40], Amycolatopsis orientalis [20], and A. mediterranei [39].

As is common with serine integrase-mediated recombination, the $a t t B$ sites in $A$. marina are located within open reading frames and potentially disrupt the gene. The TG1 attB ${ }^{A m}$ site is located within a gene predicted to encode a putative succinyldiaminopimelate transaminase (WP_091671332.1), and the R4 attB ${ }^{A m}$ site is located within a gene predicted to code for a fatty-acyl-CoA synthase (SFB62308.1). Compared to the wild-type (unintegrated) strain, the strains with integrated pHG4 or pJH1R4 did not show any difference in 
Table 2 The original $a t t B$ sites for integrases and results of BLAST search

$\begin{array}{llll}\text { Integrase } \quad a t t B \text { sites and the best hit from BLAST } & \text { Homology } & E \text {-value }\end{array}$

(\%)

\begin{tabular}{|c|c|c|c|}
\hline$\phi 31$ & 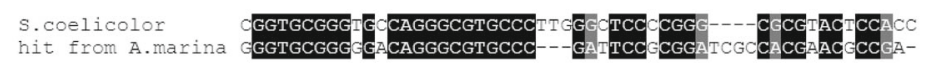 & 41 & 0.015 \\
\hline фJoe & 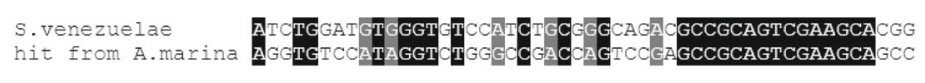 & 30 & 0.60 \\
\hline Bxb1 & 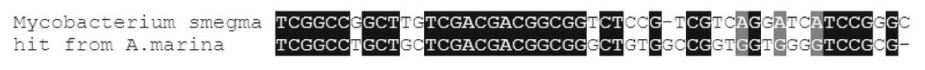 & 48 & 0.014 \\
\hline R4 & 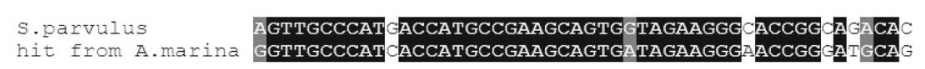 & 84 & $3 e-11$ \\
\hline
\end{tabular}

SPBC

No hit

\begin{tabular}{|c|c|c|c|}
\hline SV1 & 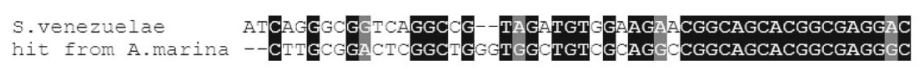 & 32 & 0.17 \\
\hline TG1 & 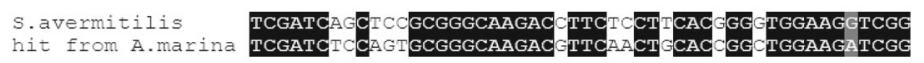 & 62 & 0.001 \\
\hline TP901 & 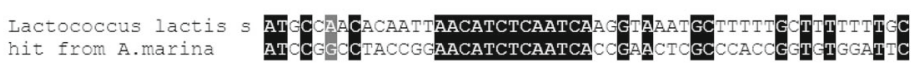 & 25 & 8.1 \\
\hline
\end{tabular}

growth. However, further study is required to investigate the effects of TG1 or R4 plasmid recombination on both primary and secondary metabolism as, for example, the integration of $\phi C 31$ integrase-based plasmids has been shown to have pleiotropic effects on bacterial physiology [48].

Currently, the following methods have been used to establish a gene transfer system in Amycolatopsis species: protoplast transformation, direct transformation of mycelia, electroporation, electroduction, and conjugation [41]. Among them, direct transformation and electroporation are most popular. While for the conjugation methods which have been widely used in Streptomyces species, there are few publications on conjugative transfer of vectors based on serine integrases in Amycolatopsis: pSET152 based on $\phi C 31$ into $A$. japonicum MG417-CF17 (conjugation frequency $=2.4 \times 10^{4}$ exconjugants $/ 10^{8}$ spores) [19] and pDZL802 based on $\phi B T 1$ into A. mediterranei U32 $\left(4 \times 10^{3}\right.$ exconjugants $/ 10^{8}$ spores) [21]. In this study, we successfully integrated plasmids into the attB sites for TG1 and R4 integrases by conjugation, which supplements the potential gene transfer methods that could be used in the genus Amycolatopsis, broadens the applicability of gene transfer systems except for the ones based on $\phi C 31$ and $\phi B T 1$ in previous publications, and will definitely facilitate the genetic manipulation of Amycolatopsis. Although the recombination efficiencies were lower for TG1 and R4, the conjugation conditions could be further optimised to achieve better conjugation results, or the application of integration based vectors for direct transformation of mycelia could be explored since the integrative vectors, for example, pMEA100 [39] and pMEA300 [49], used in direct transformation are based on integrase and corresponding attP site as well.

\section{Conclusions}

In conclusion, we have identified highly conserved sequences of the attB sites for TG1 and R4 integrases within the genus Amycolatopsis and demonstrated their use in conjugative DNA transfer. The A. marina DSM45569 attB sites showed slightly lower recombination efficiencies in vitro than the previously identified attB sites from Streptomyces spp. However, this slight reduction is not enough by itself to explain the order of magnitude reductions in conjugation frequencies observed with A. marina compared to Streptomyces spp. (Table 3). Optimising conjugation conditions could increase the conjugation frequencies further. Alternatively, 


\section{a R4 attB sites}
A marina
A balhimycina
A_japonica
A mediterranei
A orientalis
A_rifamycinica
A rubida
A_tolypomycina
A xylanica
s-parvulus

\section{b TG1 attB sites}
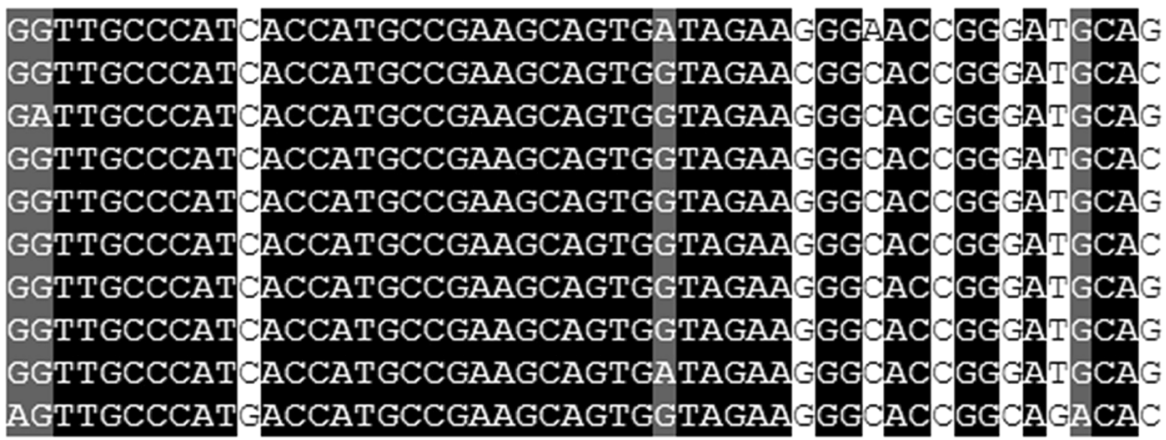
A marina
A_alba
A azurea
A balhimycina
A japonica
A lurida
A mediterranei
A-orientalis
A thermoflava
$\mathrm{S}$-avermitilis

Fig. 1 Alignment of R4 and TG1 attB sites in A. marina DSM45569 and other Amycolatopsis species. a) GenBank accession nos. of DNA sequences: Amycolatopsis balhimycina (ARBH01000005.1), Amycolatopsis japonica (NZ_CP008953.1), Amycolatopsis mediterranei (NC_022116.1), Amycolatopsis orientalis (NZ_CP016174.1), Amycolatopsis rifamycinica (NZ_JMQI01000006.1), Amycolatopsis rubida (NZ_FOWC01000001.1), Amycolatopsis tolypomycina (NZ_FNSO01000004.1), Amycolatopsis xylanica (NZ_FNON01000002.1), and S. parvulus (CP015866.1); b) GenBank accession nos. of DNA sequences: Amycolatopsis alba (NZ_KB913032.1), Amycolatopsis azurea (MUXN01000005.1), A. balhimycina (ARBH01000007.1), A. japonica (NZ_CP008953.1), Amycolatopsis lurida (FNTA01000004.1), A. mediterranei (NC_022116.1), A. orientalis (NZ_CP01674.1), Amycolatopsis thermoflava (AXBH01000004.1), and S. avermitilis (NC_003155.5)

efficiently used $a t t B$ sites for the widely used vectors, such as those based on $\phi C 31$ int/attP could be incorporated into the Amylcolatopsis genome using TG1 or R4 integrating plasmids as described here. In short, this work shows that integrative vectors are viable and promising tools for the genetic engineering of rare Actinomycetes.

\section{Methods}

Bacterial strains and culture conditions

Plasmid propagation and subcloning was conducted using E. coli Top10 (F- mcrA $\Delta$ (mrr-hsdRMS-mcrBC) \$80lacZAM15 $\triangle$ lacX74 nupG recA1 araD139 $\Delta($ ara-leu)7697 galE15 galK16 $\operatorname{rpsL}\left(\mathrm{Str}^{\mathrm{R}}\right)$ endA1 $\left.\lambda^{-}\right)$. Plasmid conjugations from $E$. coli to $A$. marina DSM45569 were carried out using E. coli ET12567(pUZ8002) containing the plasmid to be transferred as the donor [50, 51], and conjugations from E. coli to S. coelicolor and S. lividans were used as control. E. coli strains were grown in Luria-Bertani broth (LB) or on LB agar at $37^{\circ} \mathrm{C}$.
A. marina DSM45569 was purchased from the German Collection of Microorganisms and Cell Cultures (DSMZ, Germany), and maintained on SM agar plates at $30{ }^{\circ} \mathrm{C}$. Harvested spores were maintained long-term in $20 \%$ glycerol at $-80^{\circ} \mathrm{C}$. Conjugations were plated on SM agar plates containing $10 \mathrm{mM} \mathrm{MgCl}$, and ISP2 medium [52] was used for the preparation of genomic DNA [51].

\section{DNA manipulation}

E. coli transformation and gel electrophoresis were carried out as described previously [53]. Genomic DNA preparation from Streptomyces was performed following the salting out procedure in the Streptomyces manual [51]. Plasmids from E. coli were prepared using QIAprep $^{\circ}$ Spin Miniprep Kit (Qiagen, Germany) following the manufacturer's instructions. Polymerase Chain Reaction (PCR) was carried out using Phusion ${ }^{\circ}$ High-Fidelity DNA Polymerase (NEB, USA) according to the manufacturer's instructions. The primers used in this study 


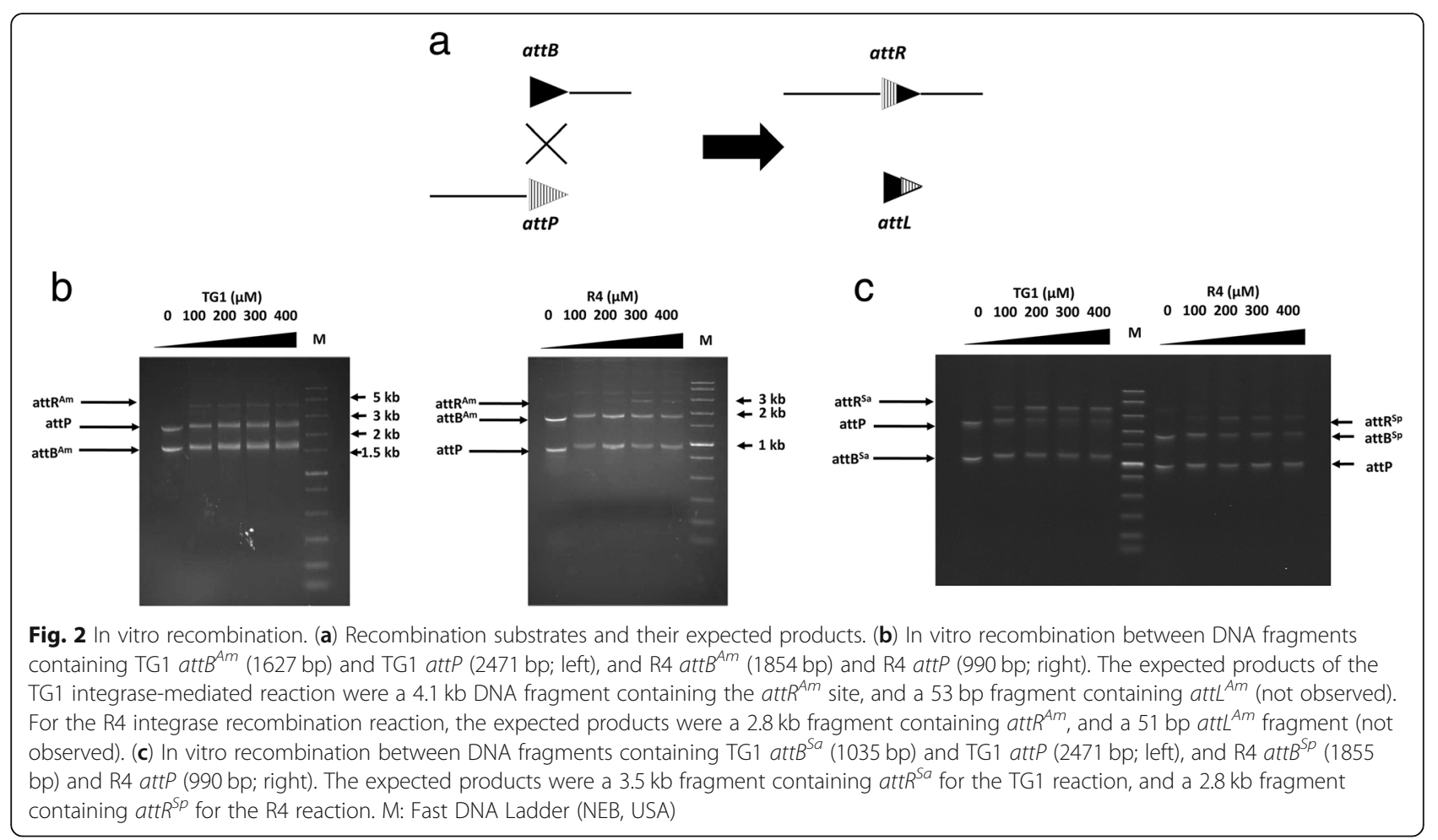

are listed in Table 1. DNA samples were purified by the QIAquick Gel Extraction Kit (Qiagen, Germany).

\section{Plasmid construction}

The integrating plasmid pHG4 contains the TG1 int/ attP locus and the apramycin-resistance gene $(\operatorname{aac}(3) I V)$ for selection (Fig. 5a). The fragment containing oriT, aac(3)IV and TG1 int/attP was amplified from plasmid pBF20 [54] using the primer pair pHG4-for/pHG4-rev. The fragment was joined via In-Fusion cloning to the 3344 bp HindIII-PacI fragment from pBF22 [54] (containing the E. coli plasmid replication origin, the bla gene encoding resistance to ampicillin and the actII-orf4/act1p expression cassette) to form the plasmid pHG4.

To construct the integrating plasmid pJH1R4 (Fig. 5a), pSET152 [55] was cut with AatII and PvuI to remove the $\phi C 31$ attP site and integrase gene. R4 phage lysate was used as the template in a PCR with the primers pJH1R4-for and pJH1R4-rev to amplify the R4 attP site and integrase coding region. The PCR product was

Table 3 Conjugation efficiency of pHG4 and pJH1R4 in different species

\begin{tabular}{lll}
\hline Exconjugants $/ 10^{8}$ spores & $\mathrm{pHG} 4$ & $\mathrm{pJH1R} 4$ \\
\hline A. marina & 160 & 20 \\
Streptomyces coelicolor & $1.47 \times 10^{3}$ & $3.28 \times 10^{4}$ \\
Streptomyces . lividans & $1.56 \times 10^{3}$ & $3.33 \times 10^{4}$ \\
\hline
\end{tabular}

joined to the AatII-PvuI fragment from pSET152 via In-Fusion cloning.

The plasmid pHG1 (Fig. 5c) was used as the template in PCR to amplify attB-containing sequences (Fig. 5d) for in vitro recombination assays. This plasmid was initially constructed for the expression of EryF. The eryF gene was amplified from Saccharopolyspora erythraea BIOT-0666 genomic DNA using the primer pair pHG1A-for/pHG1A-rev, and inserted by In-Fusion cloning into pBF20 [54] cut with NheI and PacI to form the plasmid pHG1A. The $3785 \mathrm{bp}$ fragment containing the $\phi C 31$ int/attP and hygromycin resistance gene was amplified from plasmid pBF27C [54], using the primer pair pHG1-for and pHG1-rev. Plasmid pHG1A was digested with $\mathrm{XbaI}$ and NheI, and the $5668 \mathrm{bp}$ fragment was ligated with the $3785 \mathrm{bp}$ PCR fragment from pBF27C by In-Fusion cloning to give the plasmid pHG1.

\section{In vitro recombination assays}

In vitro recombination assays were performed using PCR-amplified DNA fragments containing the attB and attP attachment sites located at the ends. Recombination between the $a t t P$ and $a t t B$ sites joined the two fragments to give a product whose length was almost the sum of the substrates (Fig. 2a). To generate the $a t t B$-containing substrates, the forward primer, TG1-attB-Am-for, contained the closest match in the A. marina genome to the characterised TG1 attB site from $S$. avermitilis, TG1 $a_{t t B}{ }^{S a}$ [30] (Fig. 1). TG1-attB-Am-for also had a 


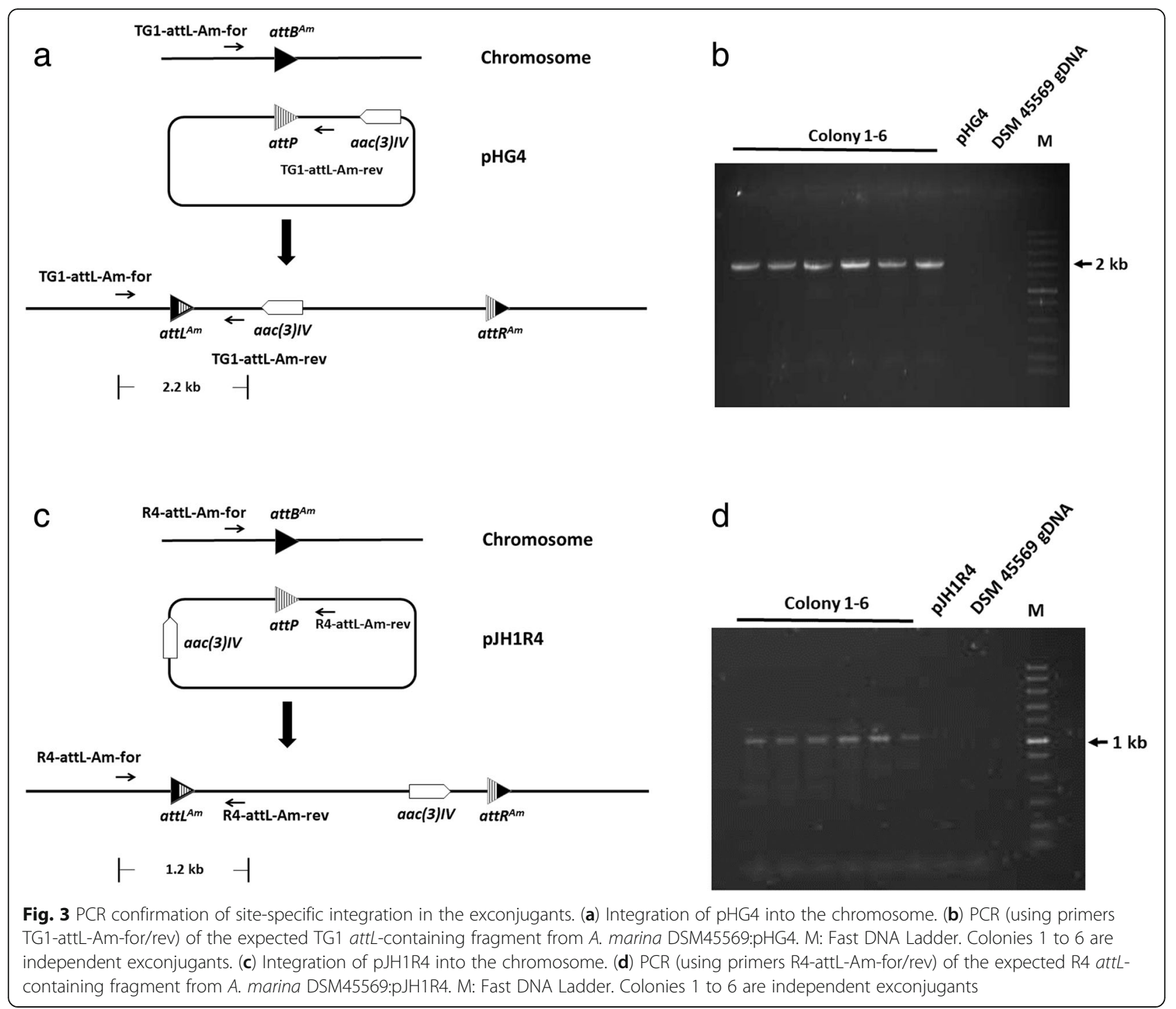

sequence identical to the 3 ' end of the actlp element from plasmid pHG1, which was used as a template for PCR (Fig. 5c). Similarly, the forward primer R4-attB-Am-for contained the closest match in the $A$. marina genome to the characterised R4 attB site from $S$. parvulus, R4 attB ${ }^{S p}$ [32] (Fig. 1). R4-attB-Am-for also had a sequence identical to the $3^{\prime}$ end of ActII-orf4 element from the template plasmid pHG1 (Fig. 5d). Forward primers TG1-attB-Sa-for and R4-attB-Sp-for were used to create positive control recombination substrates

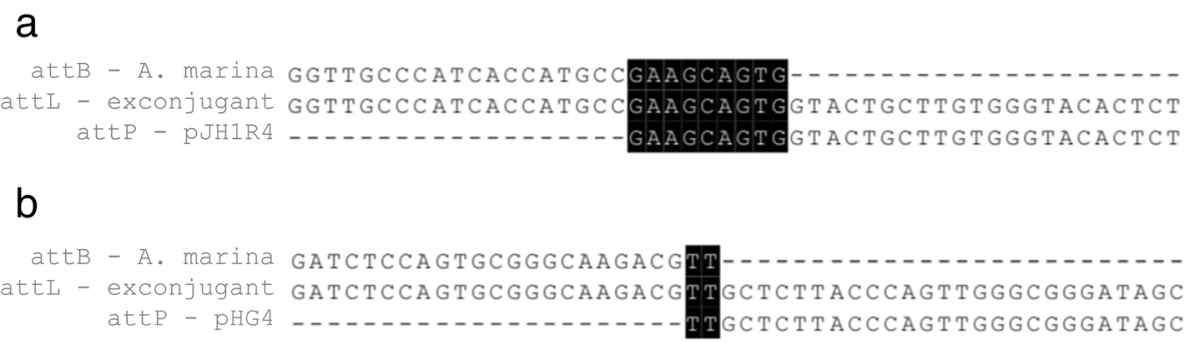

\section{b}

attB - A. marina GATCTCCAGTGCGGGCAAGACG attL - exconjugant GATCTCCAGTGCGGGCAAGACC attP - pHG4 ...................

Fig. 4 The insertion sites of R4 (a) and TG1 (b) integration plasmids in A. marina DSM45569. Sequencing (using primers R4-attL-Am-for or TG1attL-Am-for) of PCR products containing attL from exconjugants validated the site-specific recombination of the R4 and TG1 attB sites in A. marina DSM45569 after the introduction of pHG4 or pJH1R4, respectively 
a (6667 .. 6684) TG1-attP-for

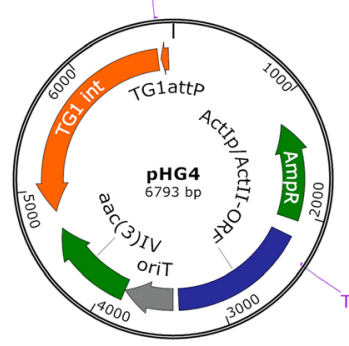

TG1-attP-rev (2324 .. 2344)

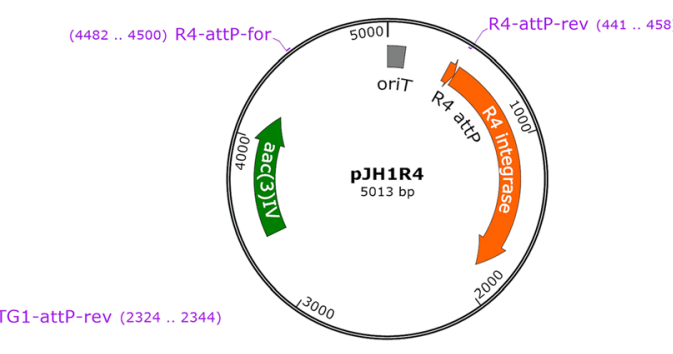

d

C

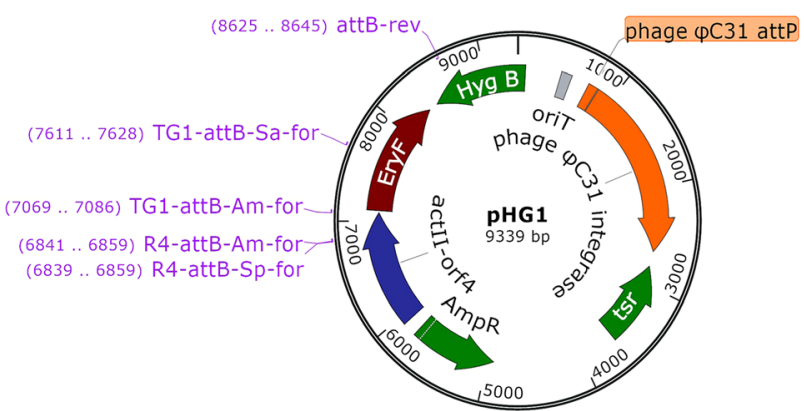

b
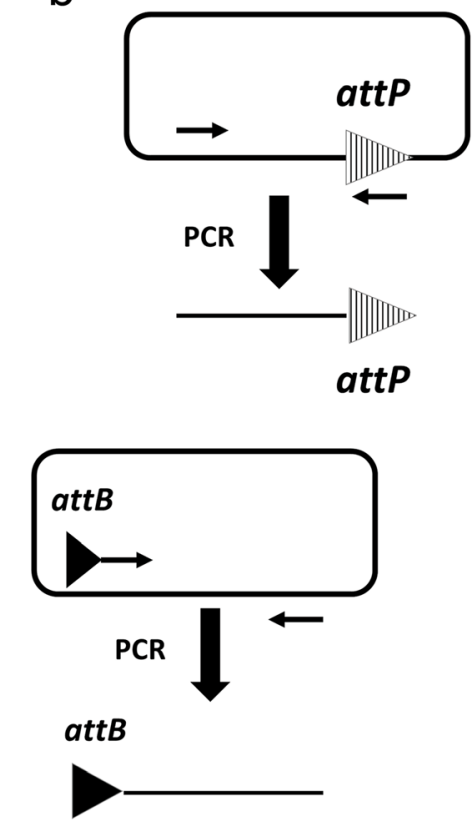

Fig. 5 Plasmids used in this study. The primer binding sites are indicated. (a) Integrating plasmids pHG4 and pJH1R4; (b) DNA substrate attPs amplified from pHG4 and pJH1R4; (c) PCR template plasmid pHG1; (d) DNA substrate attBs amplified from pHG1

containing the TG1 and R4 attB sites originally found in S. avermitilis [30] and S. parvulus [32] respectively. The reverse primer used to generate all the attB-containing substrates (attB-rev) was located within the hyg gene of pHG1; the amplified products were $1627 \mathrm{bp}$ (TG1 att$\left.B^{\mathrm{Am}}\right), 1035 \mathrm{bp}\left(\mathrm{TG} 1 \mathrm{attB}^{\mathrm{Sa}}\right), 1854 \mathrm{bp}\left(\mathrm{R} 4 a t t B^{\mathrm{Am}}\right)$ and $1855 \mathrm{bp}\left(\mathrm{R} 4 a t t B^{\mathrm{Sp}}\right)$. The DNA fragments containing the attP sites were prepared as follows; the TG1-attP fragment $(2471 \mathrm{bp})$ was amplified using the primer pair TG1-attP-for/TG1-attP-rev with pHG4 as the template, and the R4-attP fragment (990 bp) was amplified using the primer pair R4-attP-for/R4-attP-rev with pJH1R4 as the template (Fig. $5 \mathrm{~b}$ ). Note that other than the $a t t B$ and attP sites, none of the substrates contained any DNA that should interact specifically with the integrases. Moreover, each fragment was designed to be easily identifiable by molecular weight.

The integrases were purified as described previously $[27,56]$. All recombination reactions were in $20 \mu$ final volume. Recombination reactions of TG1 substrates were carried out in TG1 RxE buffer $(20 \mathrm{mM}$ Tris [pH 7.5], $25 \mathrm{mM} \mathrm{NaCl}, 1 \mathrm{mM}$ dithiothreitol [DTT], $10 \mathrm{mM}$ spermidine, $10 \mathrm{mM}$ EDTA, $0.1 \mathrm{mg} / \mathrm{ml}$ bovine serum albumin [BSA]) [57], and recombination reactions of $\mathrm{R} 4$ substrates were carried out in buffer containing $20 \mathrm{mM}$ Tris- $\mathrm{HCl}$ (pH 7.5), $50 \mathrm{mM} \mathrm{NaCl}, 10 \mathrm{mM}$ spermidine, 5 $\mathrm{mM} \mathrm{CaCl} 2$ and $50 \mathrm{mM}$ DTT [27]. Integrase was added at the concentrations indicated. Recombination substrates were used at $50 \mathrm{ng}$ each per reaction. Reactions were incubated at $30^{\circ} \mathrm{C}$ overnight and then heated $\left(10 \mathrm{~min}, 75^{\circ} \mathrm{C}\right)$ to denature integrase. The reaction mixtures were loaded on a $0.8 \%$ agarose gel in Tris/Borate/ EDTA (TBE) buffer ( $90 \mathrm{mM}$ Tris base, $90 \mathrm{mM}$ boric acid and 2 mM EDTA) containing ethidium bromide for electrophoretic separation.

\section{Abbreviations}

BSA: Bovine serum albumin; PCR: Polymerase chain reaction;

RDF: Recombination directionality factor; SM: Soya Mannitol; TBE: Tris/Borate/ EDTA

\section{Acknowledgements}

Not applicable.

\section{Funding}

This work was supported by the Biotechnology and Biological Sciences Research Council project grant BB/K003356/1, and Buvani Murugesan acknowledges the receipt of a summer studentship from the Department of Biology, University of York. These funding organisations were not involved in the design of the study and collection, analysis, and interpretation of data, or in writing the manuscript.

\section{Availability of data and materials}

All data generated or analysed during this study are included in this published article.

\section{Authors' contributions}

HG designed the study, performed the experiments and wrote the manuscript. BM participated in TG1 experiments. JH constructed pJH1R4 and purified R4 integrase. SKE purified TG1 integrase. WMS and MCMS revised the manuscript. All authors read and approved the final manuscript. 


\section{Ethics approval and consent to participate}

Not applicable.

\section{Consent for publication}

Not applicable.

\section{Competing interests}

The authors declare that they have no competing interests.

\section{Publisher's Note}

Springer Nature remains neutral with regard to jurisdictional claims in published maps and institutional affiliations.

\section{Author details}

'Department of Biology, University of York, York, North Yorkshire YO10 5DD, UK. ${ }^{2}$ Institute of Molecular, Cell and Systems Biology, University of Glasgow, Glasgow G12 8QQ, UK. ${ }^{3}$ Present address: School of Science, Engineering \&

Design, Teesside University, Middlesbrough TS1 3BX, UK.

\section{Received: 9 December 2018 Accepted: 30 April 2019}

\section{Published online: 04 June 2019}

\section{References}

1. Jones SE, Ho L, Rees CA, Hill JE, Nodwell JR, Elliot MA. Streptomyces exploration is triggered by fungal interactions and volatile signals. Elife. 2017;6:e21738.

2. Zarins-Tutt JS, Barberi TT, Gao H, Mearns-Spragg A, Zhang L, Newman DJ, Goss RJM. Prospecting for new bacterial metabolites: a glossary of approaches for inducing, activating and upregulating the biosynthesis of bacterial cryptic or silent natural products. Nat Prod Rep. 2016;33(1):54-72.

3. Jose PA, Jebakumar SRD. Non-streptomycete actinomycetes nourish the current microbial antibiotic drug discovery. Front Microbiol. 2013;4:240.

4. Jung HM, Kim SY, Moon HJ, Oh DK, Lee JK. Optimization of culture conditions and scale-up to pilot and plant scales for vancomycin production by Amycolatopsis orientalis. Appl Microbiol Biotechnol. 2007; 77(4):789-95.

5. Li C, Liu X, Lei C, Yan H, Shao Z, Wang Y, Zhao G, Wang J, Ding X. RifZ (AMED_0655) is a pathway-specific regulator for rifamycin biosynthesis in Amycolatopsis mediterranei. J Appl Environ Microbiol. 2017;83(8):e03201-16.

6. Xu X, Han L, Zhao L, Chen X, Miao C, Hu L, Huang X, Chen Y, Li Y. Echinosporin antibiotics isolated from Amycolatopsis strain and their antifungal activity against root-rot pathogens of the Panax notoginseng. Folia Microbiol. 2018;64(2):171-5.

7. Hashizume H, lijima K, Yamashita K, Kimura T, S-i W, Sawa R, Igarashi M. Valgamicin C, a novel cyclic depsipeptide containing the unusual amino acid cleonine, and related valgamicins $\mathrm{a}$, $\mathrm{T}$ and $\mathrm{V}$ produced by Amycolatopsis sp. ML1-hF4. J Antibiot. 2018;71(1):129-34.

8. Li X, Wu X, Zhu J, Shen Y. Amexanthomycins A-J, pentangular polyphenols produced by Amycolatopsis mediterranei S699 $\triangle$ rifA. Appl Microbiol Biotechnol. 2018;102(2):689-702.

9. Adamek M, Alanjary M, Sales-Ortells H, Goodfellow M, Bull AT, Winkler A, Wibberg D, Kalinowski J, Ziemert N. Comparative genomics reveals phylogenetic distribution patterns of secondary metabolites in Amycolatopsis species. BMC Genomics. 2018;19(1):426.

10. Fogg PC, Colloms S, Rosser S, Stark M, Smith MC. New applications for phage integrases. J Mol Biol. 2014;426(15):2703-16.

11. Colloms SD, Merrick CA, Olorunniji FJ, Stark WM, Smith MC, Osbourn A, Keasling JD, Rosser SJ. Rapid metabolic pathway assembly and modification using serine integrase site-specific recombination. Nucleic Acids Res. 2013; 42(4):e23.

12. Smith MC. Phage-encoded serine integrases and other large serine recombinases. In: Craig NL, Chandler M, Gellert M, Lambowitz AM, Rice PA, Sandmeyer SB, editors. Mobile DNA III. Washington, DC: ASM Press; 2015. p. 253-72.

13. Saha S, Zhang W, Zhang G, Zhu Y, Chen Y, Liu W, Yuan C, Zhang Q, Zhang $\mathrm{H}$, Zhang L. Activation and characterization of a cryptic gene cluster reveals a cyclization cascade for polycyclic tetramate macrolactams. Chem Sci. 2017:8(2):1607-12

14. Sosio M, Giusino F, Cappellano C, Bossi E, Puglia AM, Donadio S. Artificial chromosomes for antibiotic-producing actinomycetes. Nat Biotechnol. 2000; 18(3):343-5.
15. Hong Y, Hondalus MK. Site-specific integration of Streptomyces $\Phi C 31$ integrase-based vectors in the chromosome of Rhodococcus equi. FEMS Microbiol Lett. 2008;287(1):63-8.

16. Thorpe HM, Smith MC. In vitro site-specific integration of bacteriophage DNA catalyzed by a recombinase of the resolvase/invertase family. Proc Natl Acad Sci U S A. 1998:95(10):5505-10.

17. Rutherford K, Van Duyne GD. The ins and outs of serine integrase sitespecific recombination. Curr Opin Struct Biol. 2014;24:125-31.

18. Baltz RH. Streptomyces temperate bacteriophage integration systems for stable genetic engineering of actinomycetes (and other organisms). J Ind Microbiol Biotechnol. 2012;39(5):661-72

19. Stegmann E, Pelzer S, Wilken K, Wohlleben W. Development of three different gene cloning systems for genetic investigation of the new species Amycolatopsis japonicum MG417-CF17, the ethylenediaminedisuccinic acid producer. J Biotechnol. 2001;92(2):195-204.

20. Lei X, Zhang C, Jiang Z, Li X, Shi Y, Liu M, Xie Y, Wang L, Hong B. Complete genome sequence of Amycolatopsis orientalis CPCC200066, the producer of norvancomycin. J Biotechnol. 2017;247:6-10.

21. Li C, Zhou L, Wang Y, Zhao G, Ding X. Conjugation of $\varphi B T 1$-derived integrative plasmid pDZL802 in Amycolatopsis mediterranei U32. Bioengineered. 2017;8(5):549-54.

22. Bian J, Li Y, Wang J, Song FH, Liu M, Dai HQ, Ren B, Gao H, Hu X, Liu ZH. Amycolatopsis marina sp. nov., an actinomycete isolated from an ocean sediment. Int J Syst Evol Microbiol. 2009;59(3):477-81.

23. Romano S, Jackson S, Patry S, Dobson A. Extending the "one strain many compounds"(OSMAC) principle to marine microorganisms. Mar Drugs. 2018; 16(7):244.

24. Combes P, Till R, Bee S, Smith MC. The Streptomyces genome contains multiple pseudo-attB sites for the $\varphi(31$-encoded site-specific recombination system. J Bacteriol. 2002;184(20):5746-52.

25. Fogg PC, Haley JA, Stark WM, Smith MC. Genome integration and excision by a new Streptomyces bacteriophage, $\varphi$ Joe. Appl Environ Microbiol. 2016; 83(5):e02767-16.

26. Ghosh P, Pannunzio NR, Hatfull GF. Synapsis in phage Bxb1 integration: selection mechanism for the correct pair of recombination sites. J Mol Biol. 2005:349(2):331-48.

27. Takamasa M, Yayoi H, Yang YZ, Tomoyasu N, Munehiko A, Hideo T, Makoto S. In vivo and in vitro characterization of site-specific recombination of actinophage R4 integrase. J Gen Appl Microbiol. 2011 57(1):45-57.

28. Lazarevic V, Düsterhöft A, Soldo B, Hilbert H, Mauel C, Karamata D. Nucleotide sequence of the Bacillus subtilis temperate bacteriophage SP $\beta C 2$. Microbiology. 1999;145(5):1055-67.

29. Fayed B, Younger E, Taylor G, Smith MC. A novel Streptomyces spp. integration vector derived from the $S$. venezuelae phage, SV1. BMC Biotechnol. 2014;14(1):51.

30. Morita K, Yamamoto T, Fusada N, Komatsu M, Ikeda H, Hirano N, Takahashi $H$. In vitro characterization of the site-specific recombination system based on actinophage TG1 integrase. Mol Gen Genomics. 2009;282:607-16.

31. Christiansen B, Johnsen M, Stenby E, Vogensen F, Hammer K. Characterization of the lactococcal temperate phage TP901-1 and its sitespecific integration. J Bacteriol. 1994;176(4):1069-76.

32. Shirai M, Nara $H$, Sato A, Aida T, Takahashi H. Site-specific integration of the actinophage R4 genome into the chromosome of Streptomyces parvulus upon lysogenization. J Bacteriol. 1991;173(13):4237-9.

33. Li X, Zhou $X$, Deng Z. Vector systems allowing efficient autonomous or integrative gene cloning in Micromonospora sp. strain 40027. Appl Environ Microbiol. 2003;69(6):3144-51.

34. Marcone GL, Carrano L, Marinelli F, Beltrametti F. Protoplast preparation and reversion to the normal filamentous growth in antibiotic-producing uncommon actinomycetes. J Antibiot. 2010;63:83-8.

35. Wolf T, Gren T, Thieme E, Wibberg D, Zemke T, Pühler A, Kalinowski J. Targeted genome editing in the rare actinomycete Actinoplanes sp. SE50/ 110 by using the CRISPR/Cas9 system. J Biotechnol. 2016:231:122-8.

36. Wagner N, Oßwald C, Biener R, Schwartz D. Comparative analysis of transcriptional activities of heterologous promoters in the rare actinomycete Actinoplanes friuliensis. J Biotechnol. 2009;142:200-4.

37. Anzai Y, lizaka Y, Li W, Idemoto N, Tsukada SI, Koike K, Kinoshita K, Kato F. Production of rosamicin derivatives in Micromonospora rosaria by introduction of $d$-mycinose biosynthetic gene with ФC31-derived integration vector pSET152. J Ind Microbiol Biotechnol. 2009;36:1013-21. 
38. Kim DY, Huang YI, Choi SU. Cloning of metK from Actinoplanes teichomyceticus ATCC31121 and effect of its high expression on antibiotic production. J Microbiol Biotechnol. 2011;21(12):1294-8.

39. Madoń J, Hütter R. Transformation system for Amycolatopsis (Nocardia) mediterranei: direct transformation of mycelium with plasmid DNA. J Bacteriol. 1991;173(20):6325-31.

40. Kilian R, Frasch H-J, Kulik A, Wohlleben W, Stegmann E. The VanRS homologous two-component system VnIRSAb of the glycopeptide producer Amycolatopsis balhimycina activates transcription of the vanHAXSC genes in Streptomyces coelicolor, but not in A. balhimycina. Microb Drug Resist. 2016;22(6):499-509.

41. Malhotra S, Lal R. The genus Amycolatopsis: indigenous plasmids, cloning vectors and gene transfer systems. Indian J Microbiol. 2007;47(1):3-14.

42. Kumari R, Singh P, Lal R. Genetics and genomics of the genus Amycolatopsis. Indian J Microbiol. 2016;56(3):233-46.

43. Shen Y, Huang H, Zhu L, Luo M, Chen D. Type II thioesterase gene (ECOorf27) from Amycolatopsis orientalis influences production of the polyketide antibiotic, ECO-0501 (LW01). Biotechnol Lett. 2012;34(11):2087-91.

44. Lee K, Lee B, Ryu J, Kim D, Kim Y, Lim S. Increased vancomycin production by overexpression of MbtH-like protein in Amycolatopsis orientalis KFCC 10990P. Lett Appl Microbiol. 2016;63(3):222-8.

45. Mandali S, Dhar G, Avliyakulov NK, Haykinson MJ, Johnson RC. The sitespecific integration reaction of Listeria phage A118 integrase, a serine recombinase. Mob DNA. 2013;4(2).

46. Yoon B, Kim I, Nam JA, Chang HI, Ha CH. In vivo and in vitro characterization of site-specific recombination of a novel serine integrase from the temperate phage EFC-1. Biochem Biophys Res Commun. 2016;473:336-41.

47. Yang L, Nielsen AAK, Fernandez-Rodriguez J, McClune CJ, Laub MT, Lu TK, Voigt CA. Permanent genetic memory with> 1-byte capacity. Nat Methods. 2014;11:1261-6.

48. Talà A, Damiano F, Gallo G, Pinatel E, Calcagnile M, Testini M, Fico D, Rizzo D, Sutera A, Renzone G. Pirin: a novel redox-sensitive modulator of primary and secondary metabolism in Streptomyces. Metab Eng. 2018;48:254-68.

49. Vrijbloed J, Madoń J, Dijkhuizen L. Transformation of the methylotrophic actinomycete Amycolatopis methanolica with plasmid DNA: stimulatory effect of a pMEA300-encoded gene. Plasmid. 1995;34(2):96-104.

50. MacNeil D. Characterization of a unique methyl-specific restriction system in Streptomyces avermitilis. J Bacteriol. 1988;170(12):5607-12.

51. Kieser T, Bibb M, Buttner M, Chater K, Hopwood D. Practical Streptomyces genetics. Norwich: the John Innes Foundation; 2000.

52. Shirling E, Gottlieb D. Methods for characterization of Streptomyces species. Int J Syst Evol Microbiol. 1966;16(3):313-40.

53. Sambrook J, Russell DW. Molecular cloning: a laboratory manual. New York: Cold Spring Harbor Laboratory Press; 2001.

54. Fayed B, Ashford DA, Hashem AM, Amin MA, El Gazayerly ON, Gregory MA, Smith MC. Multiplexed integrating plasmids for engineering the erythromycin gene cluster for expression in Streptomyces and combinatorial biosynthesis. Appl Environ Microbiol. 2015;81(24):8402-13.

55. Wilkinson CJ, Hughes-Thomas ZA, Martin CJ, Bohm I, Mironenko T, Deacon M, Wheatcroft M, Wirtz G, Staunton J, Leadlay PF. Increasing the efficiency of heterologous promoters in actinomycetes. J Mol Microbiol Biotechnol. 2002:4(4):417-26.

56. Rowley P, Smith M, Younger E, Smith MC. A motif in the C-terminal domain of $\varphi C 31$ integrase controls the directionality of recombination. Nucleic Acids Res. 2008;36(12):3879-91.

57. Morita K, Morimura K, Fusada N, Komatsu M, Ikeda H, Hirano N, Takahashi H. Site-specific genome integration in alphaproteobacteria mediated by TG1 integrase. Appl Microbiol Biotechnol. 2012;93(1):295-304.

Ready to submit your research? Choose BMC and benefit from:

- fast, convenient online submission

- thorough peer review by experienced researchers in your field

- rapid publication on acceptance

- support for research data, including large and complex data types

- gold Open Access which fosters wider collaboration and increased citations

- maximum visibility for your research: over $100 \mathrm{M}$ website views per year

At BMC, research is always in progress.

Learn more biomedcentral.com/submissions 